\title{
Potential sexing of spermatozoa using new antibodies to sperm surface proteins
}

\author{
JM Morrell ${ }^{1}$, A Lundequist ${ }^{2}$, M Wallgren ${ }^{3}$, I Cumming ${ }^{4}$ \\ Division of Reproduction, Swedish University of Agricultural Sciences (SLU), Uppsala, Sweden; ${ }^{2}$ Section for \\ Biochemistry, SLU; ${ }^{1,3}$ Quality Genetics, Sweden, ${ }^{4}$ Cattle Logic Ltd, UK
}

Current husbandry practices in the pig industry favour same-sex groups on welfare grounds (to prevent fighting) and because of a more even live-weight gain, with all members of the group reaching slaughter weight within a specified time. However, if male pigs are left entire, the meat develops "boar taint" as the animal reaches sexual maturity, which is considered undesirable by some consumers. Furthermore, there may be husbandry problems because of fighting. Therefore, young males must be castrated, which has attendant welfare considerations. An alternative to surgical castration would be to produce mainly female piglets by using sexed sperm doses for artificial insemination. Thus far, the only method of sexing spermatozoa which has been shown to work reliably is that of selection and separation of spermatozoa whose DNA is stained with a bis-benzimidazole dye, H33342 (Keeler et al, 1983), using the sorting capacity of a flow cytometer (Morrell et al, 1988; Johnson et al, 1989). However, the process of sorting sufficient numbers for an insemination dose in the flow cytometer takes too long since the stained spermatozoa must pass one at a time through a laser beam for detection of their DNA content. Although flow cytometric sexing of spermatozoa is $70-90 \%$ reliable, the method is slow and expensive, and sperm fertility may be reduced.

An alternative method of sperm sexing could be to use antibodies to sperm surface proteins, such as those produced by Cattle Logic Ltd (UK). The objective of the present study was to determine whether spermatozoa can be aggregated by antibody to sperm surface protein 1 and then separated into sub-populations enriched for either X- or Y-chromosome bearing spermatozoa.

The experiment was conducted in three consecutive stages: i) Antibody to a sperm surface protein 1 (Antibody 1; Cattle Logic Ltd, UK) was added to extended boar semen at a sperm concentration of $50 \times 10^{6}$ spermatozoa/mL to see if agglutination of the spermatozoa occurred. ii) Boar sperm samples treated with different concentrations of antibody 1 were either allowed to precipitate and the supernatant was removed ("fall down"), or the samples were centrifuged at 100 or $200 \mathrm{~g}$ to pellet all the spermatozoa and non-aggregated spermatozoa were allowed to swim-up from the sperm pellet into fresh extender ("swim-up"). The X:Y ratio was measured by quantitative polymerase chain reaction (qPCR). iii) Using the optimum concentration of antibody 1, the experiment was repeated, analyzing the $X: Y$ ratio in the sperm pellets and subsequently in the supernatant by qPCR. In all cases, controls (non-antibody treated semen from the same boars) were included. To determine the $\mathrm{X}$ :Y ratio in the separated sperm, SYBR-green based quantitative real-time PCR was performed Genomic DNA isolated from sperm samples was analyzed using primers designed to amplify the chromosome X-linked gene ProteoLipid Protein 1 or the chromosome Y-linked gene Sex-determining Region Y.. To calculate the percentage of X-bearing versus Y-bearing spermatozoa accurately, the cycle threshold for individual samples was compared to a standard curve generated from a serial dilution of a plasmid encoding the genes of interest.

The following results were obtained: i) Aggregation of boar spermatozoa was obtained with Antibody 1 . Aggregated spermatozoa could be roughly separated from non-aggregated spermatozoa by allowing the sperm suspension to stand at room temperature for $30 \mathrm{mins}$, enabling the aggregated spermatozoa to sink to the bottom of the tube ("fall down"). A few non-aggregated spermatozoa were present with the aggregated spermatozoa and vice versa, hence the description "roughly". When 
examined using a phase contrast microscope, the aggregation was seen to consist of large numbers of spermatozoa tightly clumped together whereas in control samples any spontaneous aggregation consisted of a few spermatozoa (e.g. two to six) in loose association.

ii) Better separations of aggregated and non-aggregated spermatozoa could be obtained by reducing the concentration of antibody used, but the effect was boar dependent (Table 1).

Table 1. X:Y ratios in boar spermatozoa treated with antibody to protein 1.

\begin{tabular}{lcc}
\hline Treatment & Method 1 ("fall down") & Method 2 ("swim-up") \\
& $X: Y$ & $X: Y$ \\
\hline Sperm supernatant Antibody $100 \mu 1$ & $42: 58$ & $44: 56$ \\
Sperm supernatant Antibody $50 \mu 1$ & $37: 63$ & $30: 69$ \\
Sperm supernatant Antibody $10 \mu \mathrm{l}$ & $30: 69$ & $47: 53$ \\
\hline
\end{tabular}

iii), the ratio of $X: Y$ in the sperm pellet was 78:22 for one boar after "fall down" and 75:25 for the other boar after "swim-up". When the experiment was repeated, the supernatants were analysed, giving a ratio of 35:65 for both boars after "fall down", 36:64 for one boar after "swim-up" and 31:69 for the other boar after "swim-up".

These results indicate that there is differential binding of antibody to protein 1 to spermatozoa, causing agglutination of some spermatozoa which appears to be different to spontaneously-occurring agglutination. The agglutinated and non-agglutinated sperm populations can be separated and show a change in the sex ratio in the two sub-populations. Although the changes in the sex ratio observed in the present study are indicative of an enrichment for either X or Y-chromosome bearing spermatozoa, the separation processes ("fall down" and "swim-up") were very rough. It may be possible to refine the separation process to obtain a higher degree of enrichment, for example by using colloid centrifugation with Androcoll-P.

In conclusion, following agglutination of boar spermatozoa with antibody to protein 1, it is possible to enrich the supernatant for Y-chromosome bearing boar spermatozoa and the sperm pellet for X-chromosome bearing spermatozoa. The two sperm sub-populations can be separated roughly by allowing agglutinated sperm to precipitate or non-agglutinated spermatozoa to swim-up after centrifugation, suggesting that refining the separation technique could improve the shift in $X: Y$ ratio and allow sufficient spermatozoa for an AI dose to be prepared. However, the qPCR method requires optimising for boar sperm DNA if it is to be used for quality control.

\section{Acknowledgement:}

JM Morrell was financed by the Swedish Ministry of Agriculture (Project number 31-3389/09).

\section{References}

Keeler KD, MacKenzie NM, Dresser DW 1983 Flow microfluorimetric analysis of living spermatozoa stained with Hoechst 33342. Journal of Reproduction \& Fertility 68 205-212.

Morrell JM, Keeler KD, Noakes DE, MacKenzie NM Dresser DW 1988 Sexing of sperm by flow cytometry. The Veterinary Record 122 322-324.
Johnson LA, Flook JP Hawk HW 1989 Sex preselection in rabbits: live births from $X$ and $Y$ sperm separated by DNA and cell sorting. Biology of Reproduction 41 199-203.

Van Wienen $M$, Johannisson A, Wallgren $M$, Parlevliet J, Morrell JM 2011 Single Layer Centrifugation with Androcoll ${ }^{\mathrm{TM}}-\mathrm{P}$ can be scaled-up to process larger volumes of boar semen. ISRN Veterinary Science doi:10.5402/2011/548385. 